\title{
Contribution Mechanisms and Types of Cost Threshold in the Subscription Game with Private Information on Valuation: Public Good Experiments
}

\author{
Hui-Chun Peng \\ Department of Public Finance, National Taipei University, Taiwan \\ Email: hcpeng@mail.ntpu.edu.tw
}

How to cite this paper: Peng, H. C. (2020). Contribution Mechanisms and Types of Cost Threshold in the Subscription Game with Private Information on Valuation: Public Good Experiments. Theoretical Economics Letters, 10, 1239-1252.

https://doi.org/10.4236/tel.2020.106075

Received: November 6, 2020

Accepted: December 8, 2020

Published: December 11, 2020

Copyright $\odot 2020$ by author(s) and Scientific Research Publishing Inc. This work is licensed under the Creative Commons Attribution International License (CC BY 4.0).

http://creativecommons.org/licenses/by/4.0/

\begin{abstract}
This paper conducts a lab experiment to analyze individual voluntary contribution behaviors in the simultaneous and sequential contribution mechanisms with different types of the cost threshold. The experimental results show that individual contribution behaviors are different when individuals face different types of cost threshold and participate in situations with different contribution mechanisms. When the cost threshold is certain, the contribution in the simultaneous contribution mechanism is significantly higher than the contribution in the sequential contribution mechanism. Furthermore, the contribution with cost certainty is significantly higher than the contribution with cost uncertainty, whether the contribution mechanism is a simultaneous or sequential institution. As for individual characteristic variables, the study finds that the "cooperative" individual contributes more to the public goods than the "individualistic" individual does.
\end{abstract}

\section{Keywords}

Simultaneous Contribution Mechanism, Sequential Contribution

Mechanism, Cost Threshold, Laboratory Experiment

\section{Introduction}

The mechanisms used to allocate public goods vary widely. A common approach for the provision of public goods in modern societies is voluntary private contributions. Many voluntary contribution processes are evident in contemporary societies, such as private donations, fundraising by non-profit organizations, or 
construction work funded by neighborhood associations; thus, private contributions are a part of the modern social landscape and are worthy of investigation.

This paper focuses on three important considerations that may affect individual contribution behavior. The first is contribution mechanisms. Some supermarkets use checkstand donation campaigns to support the community, for instance, but since the cashiers do not tell you how much money has been collected when you make the contribution decision, this type of contribution mechanism is called the simultaneous contribution mechanism; by contrast, if the donor knows the accumulated amounts when contributing to the public good, the process constitutes the sequential contribution mechanism. For instance, churches may announce an organ fund campaign and update the contribution level on a regular basis, or local governments might announce the seed donations to future contributors when they launch new public good projects.

The second consideration is the cost threshold. When an individual contributes to a public good, he or she may consider how much money is needed to provide the public good; that is, they consider the cost threshold. Some public projects may announce the exact cost needed to provide the public good; for example, projects posted on the Kickstarter website announce the amount of funding they would like to collect and make that information visible to potential donors. However, there are also situations in which individuals do not know the exact provision cost and face cost uncertainty when contributing to the public good, such as when the exact cost of construction work is unknown because bidding among potential contractors has not been completed, or when suppliers have limited resources to research the cost of completing a public good project. The role of cost threshold is thus an important factor that should be considered when investigating private contribution behavior.

The last consideration in this project is the valuation of the public good. Previous studies, such as Palfrey and Rosenthal (1984), Bagnoli and Lipman (1989), and Isaac et al. (1989), have been performed in information-rich environments, where the valuation of the public good is common knowledge; however, in most real-world situations, individuals have limited information about others' valuation of the public good, meaning that the valuation of the public good is usually private information. Thus, this study operates under the assumption that the valuation of the public good is private information, aligning this paper more closely with the conditions obtain in daily life.

To the best of my knowledge, no current paper considers these three factors at the same time when investigating private contributions to a discrete public good in the context of a subscription game. This paper aims to explore whether the role of cost threshold affects contribution decisions in the simultaneous and sequential contribution mechanisms. Furthermore, this paper investigates whether contribution order affects individual contribution in the sequential contribution mechanism when cost threshold uncertainty or certainty is considered.

The experiment's results demonstrate that the average group contribution in 
the simultaneous mechanism is significantly higher than the average group contribution in the sequential mechanism when the cost threshold is certain. In addition, the average group contribution with cost certainty is significantly higher than the average group contribution with cost uncertainty, whether the contribution mechanism is simultaneous or sequential institution. As for the effect of contribution order, this study finds that the last contributor makes a significantly higher contribution than the earlier contributors do when the cost threshold is certain. This paper also finds that the "cooperative" individual contributes more to the public goods than the "individualistic" individual does.

The paper is structured as follows. Section 2 summarizes the related studies that focus on the private contribution mechanism. Section 3 demonstrates the experimental design, experimental treatment, and how to conduct the lab experiment. Section 4 discusses the experimental results. This paper reports the aggregate result for group contributions first. Then, this paper analyzes the individual contribution with different contribution orders. Finally, this paper uses the individual data to examine the determinants of contributions. Section 5 is the conclusion.

\section{Literature Review}

Palfrey and Rosenthal (1984) and Bagnoli and Lipman (1989) are earlier papers that analyze private provision of the discrete public good. Both papers assumed that players make the contribution strategies in the environment with complete information of the public good valuation and a certain known cost threshold. These two theoretical papers showed the efficient provision of public goods in the subscription game may exist. Isaac et al. (1989) conducted an experiment to test the efficacy of a threshold public good mechanism where the valuation of the public good is common knowledge and the cost threshold is known. They found the full refund rule dramatically improves the provision of the public good in the environment with high and medium cost threshold.

An early theoretical analysis of the sequential contribution to a public good is provided by Varian (1994). In the model with the discrete public good and complete information of public good valuation, he found that the sequential contribution enables the early contributor to free ride off the latter one and the total contribution under the sequential institution is lower than that under the simultaneous institution. This finding asserts that the leader in a sequential public good game tries to exploit the first mover advantage and leaves the burden of providing the public good to the following contributors. Gächter et al. (2010) examined Varian's prediction via a laboratory experiment. The experimental result was consistent with the theoretical prediction that total contribution is lower under the sequential mechanism than the simultaneous alternative when contributors' preferences are sufficiently different. Bag and Roy (2011) extended Varian's model and treated donors' values of the public good as private information. They showed that the expected total contribution generated in a perfect 
Bayesian equilibrium of the sequential contribution game is at least as large as that in a Bayesian-Nash equilibrium of the simultaneous contribution game. This occurs because when donors are uncertain about other players' values of the public good, the earlier donors may be cautious in free-riding on prospective donors.

Andreoni (1998) focused on the role of seed money in a discrete public good sequential game setting. He demonstrated that adopting a sequential fundraising strategy can increase the likelihood of providing the public good when a cost threshold exists. Subsequently, Bracha et al. (2011) tested Andreoni's theory experimentally and found that the experimental results are supportive of the theory when the cost threshold is sufficiently high.

Realizing the cost threshold uncertainty may affect the individual contribution behavior, Nitzan and Romano (1990), Suleiman (1997), and McBride (2006) introduce the cost threshold uncertainty into the discrete public good model. The first two papers found the possibility of inefficient equilibrium under the threshold uncertainty. McBride (2006) investigated how the degree of the threshold uncertainty affects individual contributions and found that an increase of the threshold uncertainty in the sense of mean-preserving spread increases the individual contribution when the value of the public good is sufficiently high; otherwise, it decreases the individual contribution when the value of the public good is sufficiently low. Wit and Wilke (1998) conducted the experiment to investigate the effects of threshold uncertainty on contribution to the discrete public good. The main finding in their paper was that the cost threshold uncertainty decreases the level of cooperation only under the high uncertainty case, not under the low uncertainty case.

Gustafsson et al. (1999) conducted two experiments to compare the voluntary contribution to public goods with the same expected provision threshold but different variances. They found that subjects contribute more than the expected provision threshold, but the average contribution is smaller in the high variance group. Analyzing a similar question, Suleiman et al. (2001) showed that the effect of threshold uncertainty is moderated by the threshold mean: contribution to the public good increases as a function of uncertainty for the lower threshold mean, but decreases for the higher threshold mean.

Barbieri and Malueg (2010) and Gronberg and Peng (2014) considered both the cost threshold uncertainty and private information on valuations for a public good in a simultaneous subscription game. Barbieri and Malueg (2010) focused on whether changing the intensity and dispersion of the value distribution affects players' contribution equilibrium. They found that increasing the value distribution in the sense of first order stochastic dominance, or dispersing the value distribution in the sense of mean-preserving spread increases the contribution. Gronberg and Peng (2014) focused on the effect of changing the cost threshold distribution. They found that increasing the uncertainty level of the cost in the sense of mean-preserving spread decreases individual contribution. 


\section{Experimental Method}

\subsection{Design}

This experiment consists of three stages. In the first stage, a voluntary public good subscription game is conducted. This is the main experiment in this study. Then, a value orientation experiment is conducted in the second stage. The last stage is a risk preference experiment.

\subsubsection{The First Stage: Subscription Game}

All subjects participated in a three-player subscription game in the first stage. At the beginning, each subject was given 60 tokens as their endowment and had to decide how to allocate them. The subject must decide how many tokens they want to allocate to a public account and how many tokens to allocate to a private account. Before making the allocation decision, each subject was given the following information:

1) Their own valuation of the public good. If the total amounts in the public account reach the cost threshold, the subject receives their own valuation of the public good.

2) A range of possible valuations of the public good their group members may have. Each subject only knows that their group members' valuations of the public good are independently and randomly drawn from [0,60]. Each possible number is chosen with the equal probability.

3) The information related to the cost threshold. Depending on the treatment a subject participates in, they can know the exact cost threshold or a range of the possible cost thresholds. To receive the valuation of the public good, the total contribution in the same group must equal or exceed the cost threshold. In the treatment with the uncertain cost threshold, the exact cost threshold is not disclosed until each subject in the same group makes the decision. After all subjects make their allocation decisions, the realized cost threshold is independently and randomly drawn from the announced ranges by the computer. In the treatment with the certain cost threshold, however, the exact cost threshold is announced when subjects make the contribution decisions.

4) The accumulated amounts in the public account. If the subject participates in the treatment of the sequential mechanism, they can know how many tokens have been collected into the public account when making allocation decisions.

After making the decision, subjects were informed about the total contribution of their group, the realized cost threshold, whether the public good is provided or not, and the income they receive in the current period. Each subject's income, $\pi_{i}$, can be represented by the following equation:

$$
\pi_{i}=60-x_{i}+G_{i}\left(\sum_{j=1}^{3} x_{j}\right)+R_{i}
$$

where $x_{i}$ is subject is allocation to the public account; $G_{i}\left(\sum_{j=1}^{3} x_{j}\right)$ is income from the public good, which can be represented by 


$$
G_{i}\left(\sum_{j=1}^{3} x_{j}\right)=\left\{\begin{array}{ll}
v_{i} & \text { if } \sum_{j=1}^{3} x_{j} \geq c \\
0 & \text { if } \sum_{j=1}^{3} x_{j}<c
\end{array},\right.
$$

where $v_{i}$ is subject is valuation of public good, and $c$ is the realized cost threshold. $R_{i}$ is the refund from the public account and it can be represented by

$$
R_{i}=\left\{\begin{array}{ll}
x_{i} & \text { if } \sum_{j=1}^{3} x_{j}<c \\
0 & \text { if } \sum_{j=1}^{3} x_{j} \geq c
\end{array} .\right.
$$

\subsubsection{The Second Stage: Social Value Orientation Experiment}

The experiment conducted in the second stage strictly follows Park's (2000) experiment; the reader is referred to that study for full details of the experimental design. According to the subjects' decisions, individuals were categorized into five different types: 1) Competitive: those who want to be better off than others; 2) Individualistic: those who want to do their best for themselves; 3) Cooperative: those who try for the best for themselves and others; 4) Altruistic: those who want to do their best for others; 5) Aggressive: those who want to do worst for others.

\subsubsection{The Third Stage: Risk Attitude Experiment}

I used Dave et al. (2010) method to measure risk preference in the third stage. Each subject had to choose one of following six choice preferences:

1) $50 \%$ chance of receiving $\$ 28$ and $50 \%$ chance of receiving $\$ 28$.

2) $50 \%$ chance of receiving $\$ 24$ and $50 \%$ chance of receiving $\$ 36$.

3) 50\% chance of receiving $\$ 20$ and 50\% chance of receiving $\$ 44$.

4) $50 \%$ chance of receiving $\$ 16$ and $50 \%$ chance of receiving $\$ 52$.

5) $50 \%$ chance of receiving $\$ 12$ and $50 \%$ chance of receiving $\$ 60$.

6) $50 \%$ chance of receiving $\$ 2$ and $50 \%$ chance of receiving $\$ 70$.

To determine the earning in this stage, the subject plays the chosen gamble. The subject is classified risk-averse if he chooses 1 to 4 , risk-neutral if he chooses 5 , and risk-seeking if he chooses $6^{1}$.

\subsection{Treatments}

According to contribution mechanisms, simultaneous or sequential contribution mechanism, and the types of cost threshold, uncertain or certain cost threshold, there are 4 treatments in this experiment which are called simultaneous-certain (IC), simultaneous-uncertain (IU), sequential-certain (QC), and sequential-uncertain $(\mathrm{QU})$ treatment, respectively.

In the IC treatment, subjects make their contribution decisions at the same time. As to the cost threshold of providing the public good, subjects know the exact amount when contributing to the public good. The cost threshold is assumed to be 90 .

${ }^{1}$ The criteria and basis for this classification strictly follows Dave et al. (2010). Please refer to page 225 in Dave et al. (2010). 
In the IU treatment, subjects make their contribution decisions at the same time. However, the cost threshold is not announced when the subjects make their contribution decisions. Subjects only know that the exact cost threshold is randomly drawn from the range of 0 to 180 with the equal probability.

In the QC treatment, subjects contribute to the public good with an exogenous sequence and know the accumulated amounts of the contribution when making their contribution decisions. As to the cost threshold, it is assumed to be 90 and announced to the subjects when making the contribution decisions.

In the QU treatment, subjects follow an exogenous move to contribute to the public good. When subjects make contribution decisions, they know the accumulated amounts of the contributions. Although the accumulated amounts of contribution are announced, the exact cost threshold is not realized in this treatment. Similar to the IU treatment, subjects only know the realized cost threshold is randomly drawn from the range of 0 to 180 with the equal probability.

\subsection{Procedures}

This experiment was conducted at National Taipei University ${ }^{2}$. A total of 192 subjects volunteered to participate in this experiment. The experiment comprised 16 sessions ( 4 per treatment) with 12 subjects in each session ${ }^{3}$. Every session was conducted by $z$-tree software (Fischbacher, 2007). Each session lasted approximately 75 minutes and consisted of three stages.

At the beginning of each stage, subjects were given the instructions, which the experimenter also read aloud. The subjects knew that there were three stages in the experiment, but they did not know what they would do until the instructions were delivered at each stage. In the first stage, I conducted a between-subject design: one treatment was implemented in a single session and each subject participated in only one session and treatment. In each session, 12 players participated in 20 periods of a repeated one-shot public good game. The computer randomly and anonymously assigned the 12 players into groups of 3 and rematched them in each period. I randomly reassigned groups every period in an attempt to minimize repeated game effects and make it harder for participant reputations to develop. At the end of the 20th period, each subject drew one number randomly to determine their own payment period in the first stage. When the first stage was completed, the second and third stages were followed.

At the end of the experiment, subjects were asked to complete a short survey related to basic demographic information and were then privately paid according to their incomes earned in each stage. The conversion rate for the experiment was one token to 3 New Taiwan Dollars (NTD). Subjects earned NTD 497 on average, including a NTD 100 "show-up" payment.

${ }^{2}$ For the ease of recruiting subjects, I conduct the experiment at National Taipei University. I presume that the experimental results may not be affected by the locations.

${ }^{3}$ Because of the scarcity of experimental budget, this study only recruits 192 subjects. According to the related experimental studies, I presume that the sample size is reasonable. 


\section{Results}

The variable of interest is contribution decision. I start by reporting aggregate results for group contributions in different treatments; I then analyze individual contributions in the sequential contribution mechanisms; finally, I examine the determinants of contributions through analysis of individual data.

\subsection{Group Contributions}

Figure 1 shows the average group contribution across all 20 periods for each treatment. It is observed that, among the four treatments, the group contribution in the IC treatment is visibly highest in the greatest number of periods. The group contribution in the QU treatment declines over time, however, and is among the lowest for the four treatments in most periods. Comparing the time trends of group contribution in different treatments shows that the group contribution in the IU treatment also declines over time. Conversely, the group contributions in the QC treatment increase slightly over time.

To complement the graphical results, Table 1 presents the average group contributions, which are aggregated over all periods and all sessions in the four treatments. The average group contributions in the IC, IU, QC, and QU treatments are 70.39, 59.28, 61.00, and 49.72, respectively. According to the Kruskal-Wallis test ${ }^{4}$, the differences in group contributions among these four treatments are statistically significant $(p$-value $=0.02)$.

Next, I made pairwise comparisons of the group contributions in different treatments. By the Mann-Whitney test, I found that, when the cost threshold is certain, the group contribution in the IC treatment is significantly higher than that in the QC treatment ( $p$-value $=0.02$ ). When the cost threshold is uncertain, the Mann-Whitney test shows that the differences between group contributions in the IU and QU treatment are not statistically significant ( $p$-value $=0.15$ ).

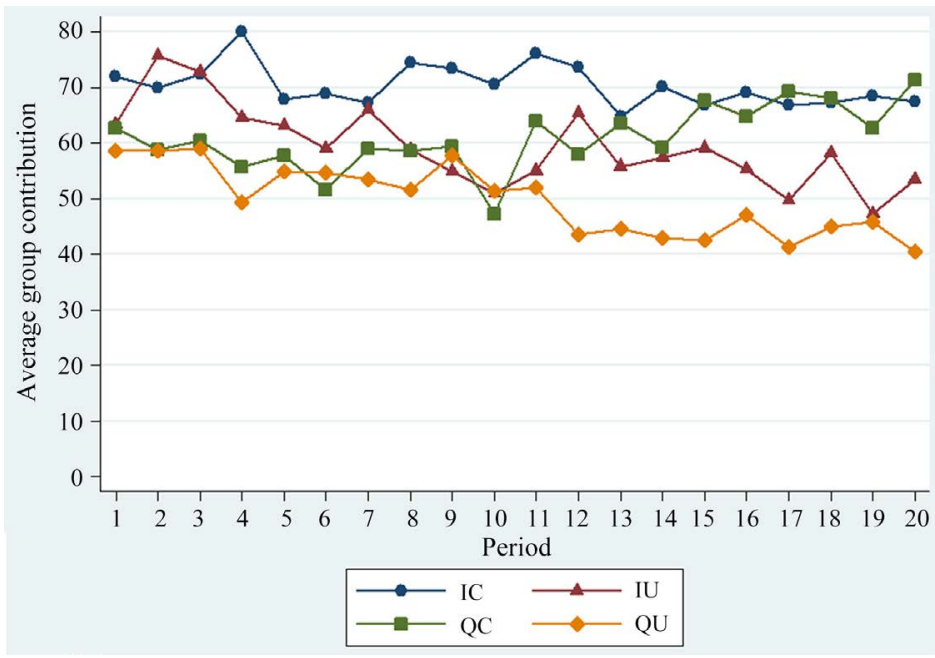

Figure 1. Average group contribution over times for each treatment.

${ }^{4}$ All non-parametric statistical tests in this paper are two-tailed. This paper uses the session-level data as an observation. 
Table 1. Average group contribution in each treatment.

\begin{tabular}{ccc}
\hline & Certain Threshold & Uncertain Threshold \\
\hline Simultaneous & 70.39 & 59.28 \\
contribution & $(1.21)$ & $(4.07)$ \\
Sequential & 61.00 & 49.72 \\
contribution & $(2.52)$ & $(2.70)$ \\
\hline
\end{tabular}

Note: Numbers in parentheses are standard deviations.

Result 1: When the cost threshold is certain, the group contribution in the simultaneous contribution mechanism is significantly higher than that in the sequential contribution mechanism.

Moreover, conducting a Mann-Whitney test on session-level data for the group contribution in the simultaneous contribution mechanism showed that the group contribution in the IC treatment is significantly higher than the group contribution in the IU treatment ( $p$-value $=0.08)$. As for the sequential contribution mechanism, the experimental results show that the group contribution in the QC treatment is significantly higher than that in the QU treatment ( $p$-value $=0.04$; Mann-Whitney test).

Result 2: Whether the contribution mechanism is simultaneous or sequential, the group contribution is significantly higher when the cost threshold is certain compared to when the cost threshold is uncertain.

\subsection{Individual Sequential Contribution}

I examined whether the contribution order affects individual contributions in the sequential contribution mechanism. Table 2 reports individual contributions with different contribution orders in the sequential contribution mechanism with certain and uncertain cost thresholds. Comparing the individual contribution in the same contribution order, but with different types of cost threshold, the Mann-Whitney test shows that the first and second contributor in the QC treatment contribute significantly more than their counterparts in the same positions in the QU treatment ( $p$-value $=0.02$ in both case); differences in the third contributor's contributions in both QC and QU treatment are not statistically significant, however $(p$-value $=1.00)$.

Looking at the QC treatment, the Kruskal-Wallis test shows that individual contributions differ as a function of contribution order ( $p$-value $=0.02)$. Furthermore, I tested the contributions of any two contribution orders with the Wilcoxon sign rank test. Comparing the first contributor's contribution with the second contributor's contribution, I find that the former, 22.18, is lower than the latter, 23.23, but the difference is not statistically significant ( $p$-value $=0.72$ ). As for comparison of the first contributor's and the third contributor's contributions, I found that the first contributor's contribution is significantly higher than the third contributor's, which was 15.59 ( $p$-value $=0.07$ ). I also found that the second contributor's contribution is significantly higher than the third contributor's ( $p$-value $=0.07$ ). The Kruskal-Wallis test shows different results when 
Table 2. Individual contributions with different contribution orders.

\begin{tabular}{cccc}
\hline & $\mathbf{1}^{\text {st }}$ contributor & $\mathbf{2}^{\text {nd }}$ contributor & $3^{\text {rd }}$ contributor \\
\hline \multirow{2}{*}{ QC Treatment } & 22.18 & 23.23 & 15.59 \\
& $(0.33)$ & $(1.40)$ & $(1.43)$ \\
QU Treatment & 17.65 & 16.16 & 15.90 \\
& $(0.83)$ & $(1.01)$ & $(1.26)$ \\
\hline
\end{tabular}

Note: Numbers in parentheses are standard deviations.

the cost threshold uncertainty is introduced into the sequential contribution mechanism, however. I found that individual contributions with different contribution orders in the QU Treatment did not differ at the level of statistical significance $(p$-value $=0.78)$.

Result 3: In the sequential mechanism with a certain threshold, the first and second contributors' average contributions are significantly higher than the third contributor's average contribution. In the sequential mechanism with an uncertain threshold, this study does not find statistically significant differences among the individual contributions across the three contributors in a group.

\subsection{Regression Analysis}

To formally examine the determinants of individual contribution decisions, I conducted random effect Tobit regressions in which the dependent variable is the individual contribution. The individual contribution is censored to lie between 0 and 60 .

I first examined the simultaneous contribution mechanism. The independent variables include a dummy variable, uncertainty, that equals 1 if the cost threshold is uncertain, and a dummy variable, success $_{t-1}$, that equals 1 if the public good is successfully provided in the previous period. I also include a continuous variable, value, which means the value of the public good that a subject can receive when the public good is successfully provided in the current period, and another variable, period, which captures the time effect.

Model (1) and (2) of Table 3 report the results in the simultaneous contribution mechanism. Focusing on the types of cost threshold first, the variable uncertainty is negative and statistically significant; this means that the individual contributes less when the cost threshold is uncertain in the simultaneous contribution mechanism. The variable value has a significant positive effect on the contribution; this shows that the higher the value of the public good a subject has, the higher the contribution they make. As for the variable period, it obtained a significant negative coefficient, indicating that an individual contributes significantly smaller amounts to the public good in the simultaneous contribution mechanism in later periods of the experiment.

Furthermore, I included some individual characteristic variables in the regression model. The effects of these control variables are shown in Model (2) of Table 3. Female is a dummy variable that is equal to 1 if the individual is a female; 
Table 3. Random effect Tobit regression results.

\begin{tabular}{|c|c|c|c|c|}
\hline & \multicolumn{2}{|c|}{ Simultaneous } & \multicolumn{2}{|c|}{ Sequential } \\
\hline & Model 1 & Model 2 & Model 3 & Model 4 \\
\hline Uncertainty & $\begin{array}{c}-3.979^{* * *} \\
(1.47)\end{array}$ & $\begin{array}{c}-3.654^{* *} \\
(1.46)\end{array}$ & $\begin{array}{c}-6.004^{* * *} \\
(1.57)\end{array}$ & $\begin{array}{c}-5.850^{* * *} \\
(1.61)\end{array}$ \\
\hline Value & $\begin{array}{c}0.671^{* * *} \\
(0.01)\end{array}$ & $\begin{array}{c}0.670^{* * *} \\
(0.01)\end{array}$ & $\begin{array}{c}0.659^{* * *} \\
(0.01)\end{array}$ & $\begin{array}{c}0.652^{* * *} \\
(0.02)\end{array}$ \\
\hline success $_{t-1}$ & $\begin{array}{c}-0.364 \\
(0.50)\end{array}$ & $\begin{array}{c}-0.183 \\
(0.52)\end{array}$ & $\begin{array}{c}-0.178 \\
(0.79)\end{array}$ & $\begin{array}{l}0.053 \\
(0.83)\end{array}$ \\
\hline Period & $\begin{array}{c}-0.220^{* * *} \\
(0.04)\end{array}$ & $\begin{array}{c}-0.213^{* * *} \\
(0.04)\end{array}$ & $\begin{array}{c}-0.002 \\
(0.05)\end{array}$ & $\begin{array}{c}-0.016 \\
(0.05)\end{array}$ \\
\hline 2nd contributor & & & $\begin{array}{l}0.348 \\
(0.87)\end{array}$ & $\begin{array}{l}0.433 \\
(0.92)\end{array}$ \\
\hline $3 r d$ contributor & & & $\begin{array}{c}-8.426^{* * *} \\
(0.90)\end{array}$ & $\begin{array}{c}-8.211^{* * *} \\
(0.94)\end{array}$ \\
\hline uncertainty $\times 2$ nd contributor & & & $\begin{array}{c}-0.991 \\
(1.25)\end{array}$ & $\begin{array}{c}-1.063 \\
(1.32)\end{array}$ \\
\hline uncertainty $\times 3 r d$ contributor & & & $\begin{array}{c}7.365^{* * *} \\
(1.27)\end{array}$ & $\begin{array}{c}7.444^{* * *} \\
(1.35)\end{array}$ \\
\hline Female & & $\begin{array}{l}2.013 \\
(1.59)\end{array}$ & & $\begin{array}{c}-0.904 \\
(1.59)\end{array}$ \\
\hline Risk-neutral & & $\begin{array}{c}-0.015 \\
(1.75)\end{array}$ & & $\begin{array}{c}-0.665 \\
(1.48)\end{array}$ \\
\hline Risk-seeking & & $\begin{array}{c}-0.074 \\
(2.01)\end{array}$ & & $\begin{array}{c}-1.071 \\
(1.75)\end{array}$ \\
\hline SVO & & $\begin{array}{l}4.666^{* *} \\
(1.94)\end{array}$ & & $\begin{array}{c}4.333^{* * *} \\
(1.58)\end{array}$ \\
\hline Constant & $\begin{array}{c}5.353^{* * *} \\
(1.20)\end{array}$ & $\begin{array}{l}3.038^{*} \\
(1.84)\end{array}$ & $\begin{array}{l}2.004 \\
(1.48)\end{array}$ & $\begin{array}{l}2.347 \\
(2.14)\end{array}$ \\
\hline \# of observation & 1824 & 1710 & 1824 & 1672 \\
\hline \# of left-censored observation & 171 & 158 & 305 & 279 \\
\hline \# of right-censored observation & 9 & 8 & 7 & 7 \\
\hline
\end{tabular}

Note: (1) Random effect Tobit regressions are with standard errors clustered on individual level. (2) Numbers in parentheses are standard errors. $(3)^{* * *}\left({ }^{* *},{ }^{*}\right)$ represents $1 \%(5 \%, 10 \%)$ significance.

males are entered as 0 . The regression result shows the gender does not have significant effect on the individual contribution in the simultaneous contribution mechanism. In the second stage of the experiment, I measured individual social value orientation. According to the experimental data, more than $90 \%$ of the subjects belong to the "individualistic" or "cooperative" categories, a result consistent with the previous studies. $S V O$ is a dummy variable used to categorize the types of individuals: if the individual is "individualistic," $S V O$ is equal to 0 ; if the individual is "cooperative", $S V O$ is equal to 1 . The results show that individuals who are "cooperative" make higher contributions to the public good than individuals who are "individualistic" do, and that this difference is statistically 
significant. In the third stage of the experiment, I measured individual risk preference. Risk-neutral and Risk-seeking are both dummy variables. I find that individual risk preference does not affect contribution decision in the simultaneous contribution mechanism.

Conducting a random effect Tobit regression in the sequential contribution mechanism, I included contribution orders in the regression model. I used 2nd contributor ( $3 \mathrm{rd}$ contributor) as a dummy variable to indicate that the subject is the second (or third) contributor in the group. I also included the interaction terms of the contribution order and uncertainty, uncertainty $\times 2$ nd contributor, and uncertainty $\times 3$ rd contributor. Similar to the analysis in the simultaneous contribution mechanism, the individual characteristic variables are considered in the regression model.

Model (3) and (4) of Table 3 show the regression results for the sequential contribution mechanism. I found that individuals also contribute significantly less when the cost threshold is uncertain in the sequential contribution mechanism. Similar to the result in the simultaneous mechanism, the higher the value of the public good, the higher the contribution a subject makes. As for the contribution order, I found that the third contributor contributes significantly less than the first contributor in the sequential contribution mechanism, but the difference is significantly smaller when the cost threshold is uncertain.

Model (4) of Table 3 illustrates that $S V O$ has a significant positive coefficient. The result shows that an individual who is "cooperative" makes significantly higher contributions to the public good than the individual who is "individualistic" does in the sequential contribution mechanism. This finding holds for both contribution mechanisms, indicating that "cooperative" individuals contribute higher amounts to the public good regardless of condition.

Result 4: The regression results provide additional evidence that, when cost threshold uncertainty exists, individuals contribute significantly smaller amounts to public goods whether the contribution mechanism is simultaneous or sequential.

\section{Conclusion}

In this paper, I conducted a laboratory experiment to analyze the voluntary individual contribution behavior in simultaneous and sequential contribution mechanisms with certain or uncertain cost thresholds. The experiment's results show that individual contribution behaviors are very different when individuals face different types of cost threshold and participate in situations with different contribution mechanisms.

When the cost threshold is certain, simultaneous contribution is significantly higher than sequential contribution. When using the simultaneous contribution mechanism, announcing the exact cost threshold may encourage individuals to contribute more to the public good. I found similar results in the case of the sequential mechanism. The experimental results provide the policy recommenda- 
tion. According to these experimental results, if the suppliers of a public good want to receive larger amounts in the form of individual contributions, they should try to adopt a simultaneous contribution mechanism and announce the exact cost threshold when the individual valuation of the public good is private information.

The participants in this study are all students. Thus, conducting experiments with non-student participants and exploring the effect of cost threshold and types of contribution mechanisms on the contribution decision of more general subjects could be a direction for future research.

\section{Acknowledgements}

This project was supported by a grant from the Ministry of Science and Technology (MOST) in Taiwan (MOST-104-2410-H-308-080). The author thanks anonymous referees' comments. The author also benefits from the comments by participants in the 2017 Western Economic Association International (WEAI) 92nd Annual Conference.

\section{Conflicts of Interest}

The author declares no conflicts of interest regarding the publication of this paper.

\section{References}

Andreoni, J. (1998). Toward a Theory of Charitable Fund-Raising. Journal of Political Economy, 106, 1186-1213. https://doi.org/10.1086/250044

Bag, P. K., \& Roy, S. (2011). On Sequential and Simultaneous Contributions under Incomplete Information. International Journal of Game Theory, 40, 119-145. https://doi.org/10.1007/s00182-010-0230-1

Bagnoli, M., \& Lipman, B. (1989). Provision of Public Goods: Fully Implementing the Core Through Private Contributions. The Review of Economic Studies, 56, 583-601. https://doi.org/10.2307/2297502

Barbieri, S., \& Malueg, D. A. (2010). Threshold Uncertainty in the Private-Information Subscription Game. Journal of Public Economics, 94, 848-861. https://doi.org/10.1016/j.jpubeco.2010.07.003

Bracha, A., Menietti, M., \& Vesterlund, L. (2011). Seeds to Succeed? Sequential Giving to Public Projects. Journal of Public Economics, 95, 416-427. https://doi.org/10.1016/j.jpubeco.2010.10.007

Dave, C., Eckel, C. C., Johnson, C. A., \& Rojas, C. (2010). Eliciting Risk Preferences: When is Simple Better? Journal of Risk and Uncertainty, 41, 219-243. https://doi.org/10.1007/s11166-010-9103-z

Fischbacher, U. (2007). z-Tree: Zurich Toolbox for Ready-Made Economic Experiments. Experimental Economics, 10, 171-178. https://doi.org/10.1007/s10683-006-9159-4

Gächter, S., Nosenzo, D., Renner, E., \& Sefton M. (2010). Sequential vs. Simultaneous Contributions to Public Goods: Experimental Evidence. Journal of Public Economics, 94, 515-522. https://doi.org/10.1016/j.jpubeco.2010.03.002

Gronberg, T. J., \& Peng, H.-C. (2014). Changes in the Threshold Uncertainty in a Simul- 
taneous Subscription Game. Theoretical Economics Letters, 4, 263-269. https://doi.org/10.4236/tel.2014.44036

Gustafsson, M., Biel, A., \& Gärling, T. (1999). Overharvesting of Resources of Unknown Size. Acta Psychologica, 103, 47-64. https://doi.org/10.1016/S0001-6918(99)00024-4

Isaac, R. M., Schmidtz, D., \& Walker, J. M. (1989). The Assurance Problem in a Laboratory Market. Public Choice, 62, 217-236. https://doi.org/10.1007/BF02337743

McBride, M. (2006). Discrete Public Goods under Threshold Uncertainty. Journal of Public Economics, 90, 1181-1199. https://doi.org/10.1016/j.jpubeco.2005.09.012

Nitzan S., \& Romano, R. E. (1990). Private Provision of a Discrete Public Good with Uncertain Cost. Journal of Public Economics, 42, 357-370. https://doi.org/10.1016/0047-2727(90)90021-9

Palfrey T., \& Rosenthal, H. (1984). Participation and the Provision of Discrete Public Goods: A Strategic Analysis. Journal of Public Economics, 24, 171-193. https://doi.org/10.1016/0047-2727(84)90023-9

Park, E. (2000). Warm-Glow versus Cold-Pickle: A Further Experimental Study of Framing Effects on Free-Riding. Journal of Economic Behavior \& Organization, 43, 405-421. https://doi.org/10.1016/S0167-2681(00)00128-1

Suleiman, R. (1997). Provision of Step-Level Public Goods under Uncertainty: A Theoretical Analysis. Rationality and Society, 9, 163-187. https://doi.org/10.1177/104346397009002002

Suleiman, R., Budesc, D. V., \& Rapoport, A. (2001). Provision of Step-Level Public Goods with Uncertain Provision Threshold and Continuous Contribution. Group Decision and Negotiation, 10, 253-274. https://doi.org/10.1023/A:1011205901283

Varian, H. R. (1994). Sequential Contributions to Public Goods. Journal of Public Economics, 53, 165-186. https://doi.org/10.1016/0047-2727(94)90019-1

Wit, A., \& Wilke, H. (1998). Public Good Provision under Environmental and Social Uncertainty. European Journal of Social Psychology, 28, 249-256. https://doi.org/10.1002/(SICI)1099-0992(199803/04)28:2<249::AID-EJSP868>3.0.CO;2-J 